\title{
Crystallization of the Photosystem II core complex and its chlorophyll binding subunit CP43 from transplastomic plants of Nicotiana tabacum
}

\author{
Dario Piano • Sabah El Alaoui • Henryk J. Korza • \\ Renata Filipek · Izabela Sabala $\cdot$ Patrycja Haniewicz $\cdot$ \\ Claudia Buechel · Daniele De Sanctis • Matthias Bochtler
}

Received: 2 July 2010/ Accepted: 21 October 2010/Published online: 10 November 2010

(c) The Author(s) 2010. This article is published with open access at Springerlink.com

\begin{abstract}
Photosystem II from transplastomic plants of Nicotiana tabacum with a hexahistidine tag at the $\mathrm{N}$-terminal end of the PsbE subunit ( $\alpha$-chain of the cytochrome $b_{559}$ ) was purified according to the protocol of Fey et al. (BBA 12:1501-1509, 2008). The protein sample was then subjected to two additional gel filtration runs in order to increase its homogeneity and to standardize the amount of detergent. Large three dimensional crystals of the core complex were obtained. Crystals of one of its chlorophyll binding subunits $(\mathrm{CP} 43)$ in isolation grew in very similar conditions that differed only in the concentration of the detergent. Diffraction of Photosystem II and CP43 crystals at various synchrotron beamlines was limited to a resolution of 7 and $14 \AA$, respectively. In both cases the diffraction quality was insufficient for an unambiguous assignment of the crystallographic lattice or space group.
\end{abstract}

D. Piano $\cdot$ S. El Alaoui $\cdot$ H. J. Korza $\cdot$ R. Filipek - I. Sabala

P. Haniewicz $\cdot$ M. Bochtler $(\bowtie)$

International Institute of Molecular and Cell Biology,

Warsaw, Poland

e-mail: mbochtler@iimcb.gov.pl; BochtlerM@cf.ac.uk

D. Piano - S. El Alaoui - H. J. Korza - R. Filipek - I. Sabala ·

P. Haniewicz $\cdot$ M. Bochtler

Max Planck Institute of Molecular Cell Biology and Genetics,

Dresden, Germany

D. Piano - D. De Sanctis

European Synchrotron Radiation Facility, Grenoble, France

C. Buechel

Institute of Molecular Biosciences, University of Frankfurt,

Frankfurt, Germany

M. Bochtler

Schools of Chemistry and Biosciences, Cardiff, UK
Keywords Photosystem II core complex · Higher plants · Nicotiana tabacum $\cdot$ CP43 $\cdot$ 3D crystallization

\begin{tabular}{|c|c|}
\hline \multicolumn{2}{|c|}{ Abbreviations } \\
\hline Bis-Tris & $\begin{array}{l}\text { Bis(2-hydroxyethyl)amino- } \\
\text { tris(hydroxymethyl)methane }\end{array}$ \\
\hline $\mathrm{CMC}$ & Critical micelle concentration \\
\hline$\alpha-\mathrm{DDM}$ & $n$-Dodecyl- $\alpha$-D-maltopyranoside \\
\hline$\beta$-DDM & $n$-Dodecyl- $\beta$-D-maltopyranoside \\
\hline$\beta$-DM & $n$-Decyl- $\beta$-D-maltopyranoside \\
\hline HT & 1,2,3-Heptanetriol \\
\hline HTG & Heptyl-D-thioglucopyranoside \\
\hline$\alpha-\mathrm{OG}$ & $n$-Octyl- $\alpha$-D-glucopyranoside \\
\hline$\beta$-OG & $n$-Octyl- $\beta$-D-glucopyranoside \\
\hline PEG & Polyethylene glycol \\
\hline PSII & Photosystem II \\
\hline Tris & Tris(hydroxymethyl)aminomethane \\
\hline$\beta$-UL & $n$-Undecyl- $\beta$-D-maltopyranoside \\
\hline$\beta$-UDTM & $n$-Undecyl- $\beta$-D-thiomaltopyranoside \\
\hline
\end{tabular}

Introduction

Photosystem II (PSII) is a multi-protein complex that consists of both membrane-embedded and soluble subunits and is one of the crucial components in oxygenic photosynthesis. It exploits the energy of light for charge separation, which ultimately drives the water splitting reaction at the manganese cluster of the complex and the transfer of electrons to plastoquinone. Several medium resolution structures are available for the PSII core complex from cyanobacteria (Kamiya and Shen 2003; Ferreira et al. 2004; Loll et al. 2005), but so far no structural data are available for PSII of higher plants. PSII complexes from cyanobacteria and 
higher plants are generally similar, but they differ with respect to light harvesting machineries (extrinsic phycobilisomes in cyanobacteria versus transmembrane light harvesting complexes in higher plants), extrinsic subunit composition (PsbU and PsbV in cyanobacteria versus subunits PsbP and PsbQ in higher plants) and ecological niche of the source organisms (thermophilic versus mesophilic) (Büchel and Kühlbrandt 2005). In this work, we report 3D crystals of PSII core complex from Nicotiana tabacum and of its CP43 subunit. Crystals were grown in very similar conditions with the PSII core complex as a starting material and diffracted to a resolution of 7 and $14 \AA$, respectively.

\section{Materials and methods}

Growth and cultivation of tobacco plants

The transplastomic plants of Nicotiana tabacum were created and described by Fey et al. (2008) and carry a hexahistidine tag sequence at the $5^{\prime}$ end of the gene coding for the PsbE subunit. The plants were kept at a constant temperature of $25^{\circ} \mathrm{C}$ and at $50 \%$ relative humidity and grown for 10-12 weeks under a light regime of $10 \mathrm{~h}$ of light and $14 \mathrm{~h}$ of darkness per day, with a light intensity of 80-100 $\mu \mathrm{mol}$ photons $/\left(\mathrm{s} \cdot \mathrm{m}^{2}\right)$. The plants were kept at a constant temperature of $25^{\circ} \mathrm{C}$ and at $50 \%$ relative humidity.

\section{PSII core complex purification}

Thylakoid membranes and Photosystem II core complex were purified as reported previously by Fey et al. (2008) with minor modifications. The Ni-NTA elution buffer (buffer A) had lower concentration of salt and higher concentration of the osmoprotectant betaine (10 mM MES $\mathrm{pH}$ 6.0, $5 \mathrm{mM} \mathrm{NaCl}, 1 \mathrm{M}$ betaine, $5 \mathrm{mM} \mathrm{CaCl}_{2}, 10 \mathrm{mM}$ $\mathrm{NaHCO}_{3}, 300 \mathrm{mM}$ imidazole, $0.03 \% \quad \beta$-DDM).

\section{Size exclusion chromatography}

The eluted PSII core complex was concentrated using Vivaspin 20 ultrafiltration membranes with $100 \mathrm{kDa}$ cutoff until a final volume of $500 \mu \mathrm{l}$ (at $0.5 \mathrm{mg} / \mathrm{ml}$ of chlorophylls). The protein sample was loaded on a gel filtration column (Superose 6 10/300 GL, GE Healthcare) equilibrated in buffer B (10 mM MES pH 6.0, $5 \mathrm{mM} \mathrm{NaCl}$, $5 \mathrm{mM} \mathrm{CaCl}_{2}, 10 \mathrm{mM} \mathrm{NaHCO}_{3}, 0.03 \% \beta$-DDM). The main peak fractions were pooled and concentrated by ultrafiltration (Vivaspin 20, $100 \mathrm{kDa}$ cutoff) to a volume of $500 \mu$. The obtained sample was subjected to a second gel filtration run and the main peak was concentrated by ultrafiltration in two steps (with Vivaspin 20, $100 \mathrm{kDa}$ cutoff, to a volume of $200 \mu \mathrm{l}$; and then with Vivaspin 500,
$30 \mathrm{kDa}$ cutoff, to a final volume of $10 \mu \mathrm{l}$ ). The chlorophyll amount in the obtained sample was determined photometrically in $80 \%$ acetone according to a protocol of Porra et al. (1989) to be around $15 \mathrm{mg} / \mathrm{ml}$.

Oxygen evolution measurements

Oxygen evolution was assessed with a Clark-type electrode (Hansatech, England) at $20^{\circ} \mathrm{C}$ in buffer B with $1 \mathrm{mM}$ 2,6-dichloro- $p$-benzoquinone and $1 \mathrm{mM}$ ferricyanide as electron acceptors in the reaction mixture.

Polyacrylamide gel electrophoresis of proteins

For denaturing SDS-PAGE, 10\% separating Tris-tricine polyacrylamide/urea gels and $4 \%$ stacking gels were used. Samples were denatured with RotiLoad (Roth) at room temperature before loading, and after the electrophoretic separation the gels were stained with Coomassie brilliant blue (Neuhoff et al. 1988) or silver (Switzer et al. 1979).

Crystallization of the PSII core complex

The core complex of $N$. tabacum PSII was crystallized using the sitting drop vapour diffusion method at $20^{\circ} \mathrm{C}$ in the dark. The conditions tested for PSII crystallization were based on the ones reported by Adir (1999) and Smatanová et al. (2007). The final volume of the crystallization drops was $10 \mu \mathrm{l}$ and they contained $1.0-1.5 \mu \mathrm{l}$ of protein sample (15 mg/ml of chlorophylls) and $2.5 \mu \mathrm{l}$ of crystallization buffer (50 mM Bis-Tris, $1 \mathrm{mM} \mathrm{CaCl}_{2}$ and 4\% PEG 4000, final concentrations). Furthermore, the detergent mixture added to the drop consisted always of two detergents: one with high and one with low CMC prepared as 5\% (w/v) stock solutions in water (Tables 1,2). Both detergents were used in a final concentration of $0.5-1 \%(w / v)$. All detergents were purchased from Anatrace, Maumee, USA. The isomeric $\mathrm{H}$ or $\mathrm{T}$ forms of the additive 1,2,3-heptanetriol (Sigma) were also prepared as a $500 \mathrm{mM}$ stock solution in water and added to the drops to a final concentration of 50-100 mM. Water was added to reach the final drops volume of $10 \mu \mathrm{l}$. First crystals appeared after 4-7 days. The reservoir buffer was composed of 10\% PEG 4000, $100 \mathrm{mM} \mathrm{NaCl}, 50 \mathrm{mM}$ Bis-Tris, pH 7.0 and used in a volume of $0.75-1 \mathrm{ml}$.

\section{Results and discussion}

PSII purification

Transplastomic N. tabacum PSII with the N-terminally histidine tagged PsbE subunit was purified according to a 
Table 1 Preliminary screening

\begin{tabular}{llll}
\hline Detergent mix & & Dominant crystal shape \\
\hline Low CMC & High CMC & \\
\hline$\beta$-DDM & $\beta$-HTG & & Group A and group B \\
$\beta$-DDM & $\beta$-OG & & Group A (needles) \\
$\beta$-DM & $\beta$-HTG & & Group A and group B \\
$\beta$-DM & $\beta$-OG & & Group A and group B \\
$\beta$-UDM & $\beta$-HTG & Group A and group B \\
$\beta$-UDM & $\beta$-OG & Group A \\
$\beta$-UDTM & $\beta$-HTG & Group A and group B \\
$\beta$-UDTM & $\beta$-OG & Group A
\end{tabular}

Influence of the detergent mixture composition on the outcome of crystallization. The detergent stock solution contained both detergents at a concentration of 5\% and was diluted tenfold in the crystallization drop. Crystal growth was monitored during the first 15 days. Group A crystals (including needle shaped crystals) appeared after 6-8 days, group B crystals appeared later

protocol reported by Fey et al. (2008). The obtained PSII sample was depleted of Light Harvesting Complex II (LHCII) impurities. In our experiments the protocol of Fey et al. (2008) was extended by two additional gel filtration steps, which increased the purity of the sample and made it possible to reduce the salt concentration in the buffer as required for crystallization trials. In the first gel filtration step, the main peak appeared inhomogeneous and was sometimes, but not always resolved into two peaks, presumably due to the monomer-dimer ratio of PSII. For the second gel filtration step, the large molecular mass fractions of the first peak were re-injected. The resulting elution profile had its maximum slightly earlier, presumably because the procedure enriched the PSII dimer (Fig. 1).

\section{Biochemical characterization}

The polypeptide composition of the purified PSII complexes was checked by SDS-PAGE (Fig. 2). The presence of the His-PsbE subunit was confirmed by western blotting

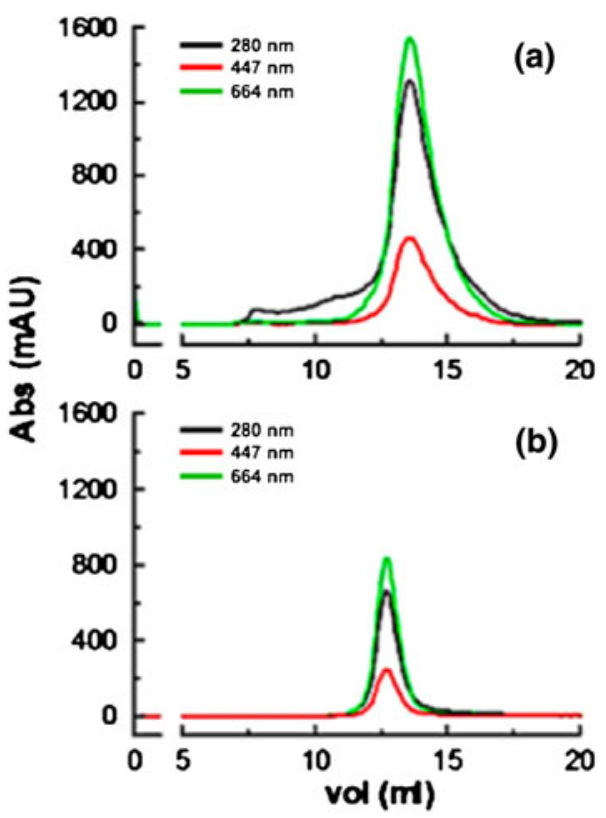

Fig. 1 Gel filtration profiles. a Profile of the first gel filtration: the protein that eluted from the Ni-NTA resin was concentrated and loaded onto the gel filtration column. The sample eluted in one main peak. The asymmetry of the peak and the high molecular mass shoulder pointed to heterogeneity of the eluted fractions. b Profile of the second gel filtration: the peak fractions of the first gel filtration were again loaded onto the same column. In the second gel filtration run, the sample eluted as a symmetric peak

with anti-His monoclonal antibodies (data not shown). Moreover, oxygen evolution was monitored. Samples were diluted in the gel filtration buffer supplemented with $1 \mathrm{M}$ betaine and $0.01 \% \beta$-DDM. The typical oxygen evolution rate was $1.2-1.4 \mathrm{mmol}_{2}$ per $\mathrm{mg}$ chlorophyll per hour.

\section{Crystallization}

Previous experiments by Adir (1999) have shown that the PSII complexes from Spinacia oleracea and Pisum sativum could be crystallized in very similar conditions. Therefore, we used the published buffer compositions in our initial

Table 2 Detailed screening

\begin{tabular}{|c|c|c|}
\hline \multicolumn{2}{|c|}{ Detergent mix* } & \multirow[t]{2}{*}{ Dominant crystal shape } \\
\hline Low CMC & High CMC & \\
\hline$\beta$-DDM & $\beta$-HTG (Sigma) & \multirow{2}{*}{$\begin{array}{l}\text { Group A and group B. Hexagonally "looking" group B grow } \\
\text { slower in the apparent unique direction than perpendicular to } \\
\text { it }\end{array}$} \\
\hline$\beta$-DDM & $\beta$-HTG (Anatrace) & \\
\hline$\alpha$-DDM & $\beta$-HTG (Anatrace) & \multirow{3}{*}{$\begin{array}{l}\text { Group A and group B. Hexagonally "looking" group B } \\
\text { crystals grow faster in the apparent unique direction than } \\
\text { perpendicular to it }\end{array}$} \\
\hline$\alpha-\mathrm{DDM}$ & $\alpha-\mathrm{OG}$ & \\
\hline$\alpha$-DDM & $\beta$-OG & \\
\hline$\beta$-DDM & $\alpha-\mathrm{OG}$ & Group A (needles) and group B \\
\hline
\end{tabular}

* Detergent mixtures selected from the screened conditions reported in Table 1. For detergent concentrations and abbreviations see Table 1 
Fig. 2 SDS-PAGE analysis of the PSII samples at different stages of purification. PSII was pooled after affinity chromatography (lanes 1 and 2, 10 and $12 \mu \mathrm{g}$, respectively), subjected to a first gel filtration step (lanes 3 and 4, 10 and $12 \mu \mathrm{g}$, respectively) and then resubjected to a second gel filtration step (lanes $5,10 \mu \mathrm{g}$ ). Lane 6 was loaded with molecular marker

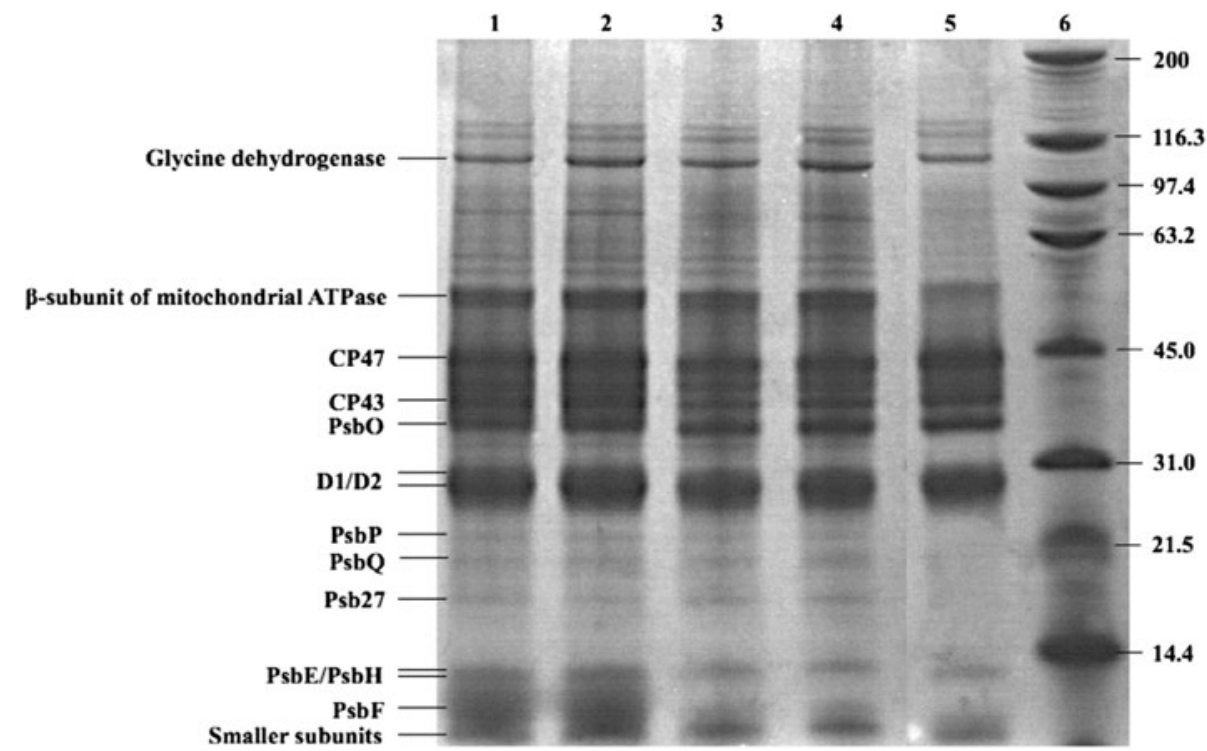

attempts to crystallize the hexahistidine tagged PSII from $N$. tabacum. As in the prior work, we used a mixture of two detergents with low and high CMCs. We tested the combinations recommended by Adir (1999), but also several other mixtures, including different anomers of alkyl maltosides and glucosides (Tables 1,2). As another important factor, Adir (1999) used the amphiphile HT as an additive in his trials. In this work, we carefully evaluated the effect of the HT on the crystallization process.

\section{Effect of HT}

HT is a mix of four stereoisomers that come in enantiomeric pairs, which are diastereomeric with respect to each other. The HT diastereomers (but not enantiomers) can be separated by melting point and are commercially available as high-melting $(\mathrm{H})$ and low-melting (T) HT fractions. The choice between the $\mathrm{H}$ and $\mathrm{T}$ fraction of $\mathrm{HT}$ affected the time of crystal growth, and also crystal shape and dimensions. The $\mathrm{H}$ fraction proved superior to the $\mathrm{T}$ fraction. The best results (with respect to the rate of crystal growth and the final crystal size) were obtained when the $\mathrm{H}$ isomers of HT was used in $0.05-0.1 \mathrm{M}$ concentration.

\section{Effect of the detergent mixture}

The use of high/low CMC detergent mixtures was found to be crucial in Adir's crystallization experiments with S. oleracea and P. sativum PSII complexes (Adir 1999). Very similar results were obtained for the $N$. tabacum PSII described here. If a single detergent was present in the drops, only spherulites could be grown. More promising crystals were grown in mixtures of $\alpha$ - or $\beta$-DDM with $\alpha$ - or $\beta$-OG (similar results were obtained if the $n$-HTG instead of OG anomers were used) (Table 2). The most successful combination contained $\alpha$-DDM and $\beta$-OG. In these conditions, at least two types of morphologically distinguishable crystals were grown. The balance between the two crystal forms depended on the amount of the detergent mixture in the crystallization drop $(0.1-2 \%)$. With $0.2-0.5 \%(\mathrm{w} / \mathrm{v})$ concentration of every component of the detergent mixture mainly group A crystals (Fig. 3) were formed after 7 days. Smaller group B crystals (Fig. 4) appeared later, after 12-15 days. An increase of the detergent concentration shifted the balance from group A to group B crystals. At the highest detergent concentrations, the growth of group A crystals was completely suppressed and only group B crystals were formed.

Analysis of group A crystals

Crystals of group A could be routinely reproduced with a mixture of $\alpha$-DDM and $\beta$-OG at a concentration $0.5 \%$ $(\mathrm{w} / \mathrm{v})$ and $50 \mathrm{mM}$ of the $\mathrm{H}$ isomers of HT. Crystals grew in 6-8 days and reached a considerable size (maximal linear dimension $0.4-0.6 \mathrm{~mm}$ ). Coomassie stained SDS-PAGE analysis of the protein mixture in the crystals showed a typical PSII core complex pattern plus the His-PsbE (Fig. 3). In order to cryoprotect crystals, a "mock" crystallization experiment without protein but with $17 \%$ PEG 400 or $22 \%$ glycerol in the usual crystallization buffer ( $1 \mathrm{mM} \mathrm{CaCl}_{2}, 50 \mathrm{mM}$ Bis-Tris, pH 7.0, 4\% PEG 4000, $0.5 \% \alpha$-DDM, $0.5 \% \beta$-OG, $50 \mathrm{mM} \mathrm{H}$ isomers of HT) was set up. Mock crystallization drops were equilibrated against the standard reservoir buffer for 1-2 days. The pretreatment of crystals in the equilibrated drops significantly reduced damage (cracking) upon their transfer to the cryobuffer. Crystals that were pretreated diffracted to a 


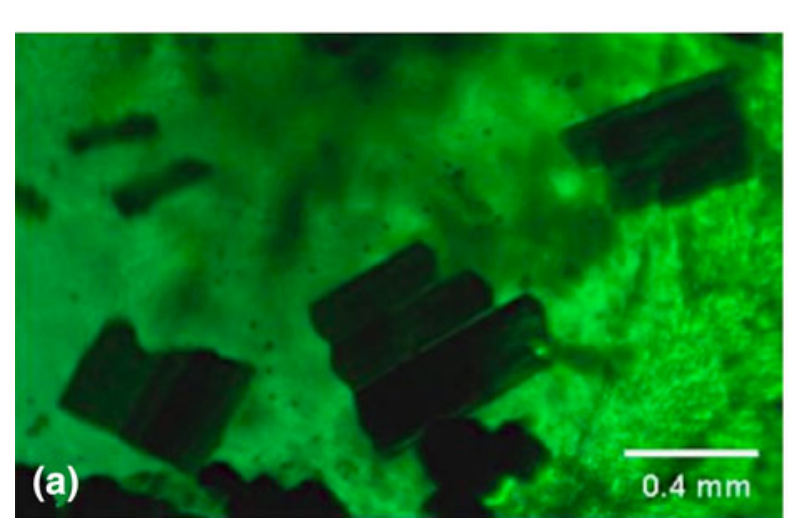

Fig. 3 Crystals of PSII core complex. a Typical morphology of crystals in the crystallization drops. b Diffraction pattern under cryogenic conditions with a limiting resolution of 7.0-7.8 $\AA$. c SDSPAGE analysis (Coomassie staining) of the protein content of the crystals. Crystals were harvested from a crystallization drop, washed extensively and dissolved in loading buffer. Lane 1 was loaded with
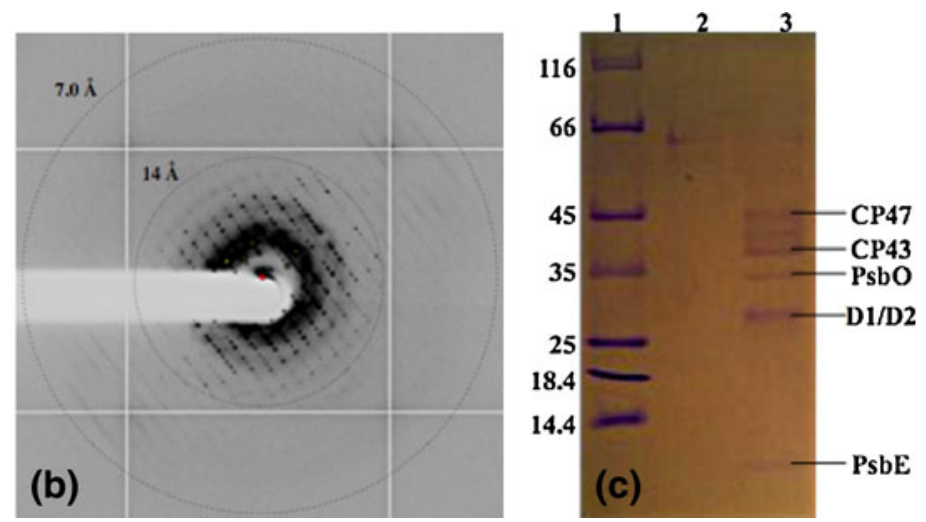

molecular marker, lane 2 with washing buffer and lane 3 with the solution containing the dissolved crystals. The complex was composed of the subunits CP47, CP43, PsbO, D1, D2 and PsbE. The subunit identification was based on the analyses of Barber et al. (1997) and Fey et al. (2008)
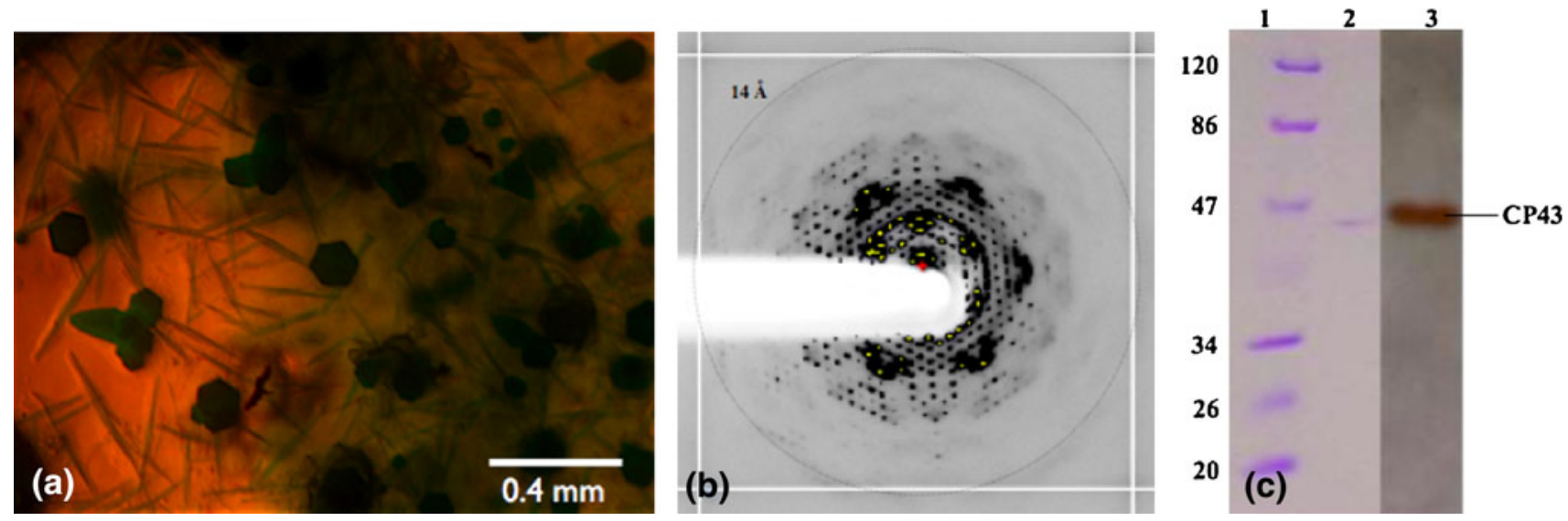

Fig. 4 Crystals of CP43. a Typical morphology of crystals in the crystallization drops. b Diffraction pattern recorded at room temperature with a limiting resolution of 12-14 $\AA$. c SDS-PAGE analysis of the protein content in the crystals. Lane 1 shows the molecular

resolution of 7.0-7.8 $\AA$ at the ESRF microfocus beamline ID23-2, Grenoble (Fig. 3).

\section{Analysis of group B crystals}

The crystals of group B appeared hexagonal with regular or irregular shape and dimensions between 0.02 and $0.2 \mathrm{~mm}$ on the hexagonal face (Fig. 4). The time of growth and crystal morphology were correlated. In the presence of a low amount of detergent, group B crystals took 6-15 days to grow and were rather irregular. In the presence of a high amount of detergent $(1-2 \% \mathrm{w} / \mathrm{v})$, crystals took only about 3 days to appear and were more regular. The final size of group B crystals was dependent on the amount of HT ( $\mathrm{H}$ isomers). When a lower amount of HT $(25 \mathrm{mM})$ was marker, lanes 2 and 3 (Coomassie and silver stained, respectively) show the protein sample obtained from the dissolved crystals after extensive washing. The observed single band was attributed to the CP43 subunit of PSII

used, crystal dimensions (across the hexagonal face) were limited to $0.01-0.05 \mathrm{~mm}$. With higher amounts of HT (50-100 mM), bigger crystals with dimensions in the $0.05-0.1 \mathrm{~mm}$ range were obtained. The protein content of group B crystals was analyzed by SDS-PAGE followed by silver staining. Only a single band was observed, which migrated slightly faster than the $45 \mathrm{kDa}$ molecular mass marker suggesting that the band represented the PSII core subunit CP43, which is known to be separable from the PSII core (Rhee et al. 1997; Büchel et al. 2000). Test exposures of the hexagonal crystals at Diamond (Didcot, UK) and at the ESRF ID23-2 (Grenoble, France) resulted in diffraction to a maximum resolution of $12-14 \AA$, but only for one orientation of the crystals. The recorded image showed features of diffuse scattering. We attributed this to 
random lattice disorder, with a short correlation length and large amplitude of displacement. Consistent with this interpretation, we observed almost no diffraction when the spindle axis was rotated by $90^{\circ}$ (Fig. 4).

\section{Conclusions}

In this work, we report the formation of two types of crystals from preparations of the PSII core complex. In the presence of a low amount of detergent mixture, crystals of the intact core complex formed first, but eventually, the CP43 core subunit alone also crystallized in the same drops. Increasing amounts of the detergents shifted the balance between the two crystal forms towards the formation of the CP43 crystals. Our findings are consistent with prior observations that the CP43 subunit can dissociate from the core complex of PSII in some conditions (Rhee et al. 1997; Büchel et al. 2000).

\section{Outlook}

Dehydration of membrane protein crystals has often improved diffraction quality. Therefore, controlled dehydration experiments were carried out on the crystal free mounting system (Kiefersauer et al. 2000) at Proteros (Martinsried, Germany) and directly at the ESRF, beamline ID14-2 (Grenoble, France). In the experiments at the synchrotron beamline, diffraction improved significantly when crystals were dehydrated from 97 to $90 \%$ relative humidity. Further dehydration did not change diffraction quality, until a drastic loss of diffraction occurred at $85 \%$ relative humidity. The diffraction could be recovered when the humidity was increased in several steps from 85 to $90 \%$ and persisted up to a relative humidity of $97 \%$. The main improvement during the dehydration steps was the appearance of diffraction spots smeared into lines up to a resolution of approximately $8 \AA$. Rehydration of the crystals tended to resolve spots, but at the expense of resolution. Protein crystallization itself is an efficient protein purification technique, and therefore we expected that crystal quality might be improved by recrystallization. Unfortunately, initial attempts with CP43 crystals were unsuccessful, because the protein precipitated when crystals were dissolved in buffer B.

Acknowledgments We are grateful to R. Kiefersauer and S. Krapp at PROTEROS, Martinsried, for the help with the initial crystal dehydration experiments. M. Nowotny kindly helped to test some crystals at synchrotron beamlines. G. Bourenkov advised on the interpretation of diffraction patterns of the CP43 crystals. H. Czapinska contributed with stimulating discussions and critically read the manuscript. We thank the staff at ESRF, Diamond, DESY and BESSY for the availability of beamtime for test exposures. This work was done with financial support from Marie Curie Host Fellowship "Transfer of Knowledge" (MTKD-CT-2006-042486) and MNiSW decision 151/6.PR UE/2007/7.

Open Access This article is distributed under the terms of the Creative Commons Attribution Noncommercial License which permits any noncommercial use, distribution, and reproduction in any medium, provided the original author(s) and source are credited.

\section{References}

Adir N (1999) Crystallization of the oxygen-evolving reaction centre of photosystem II in nine different detergent mixtures. Acta Cryst D55:891-894

Barber J, Nield J, Morris EP, Zheleva D, Hankamer B (1997) The structure, function and dynamics of photosystem two. Physiol Plant 100:817-827

Büchel C, Kühlbrandt W (2005) Structural differences in the inner part of Photosystem II between higher plants and cyanobacteria. Photosynth Res 85:3-13

Büchel C, Morris E, Barber J (2000) Crystallisation of CP43, a chlorophyll binding protein of Photosystem II: an electron microscopy analysis of molecular packing. J Struct Biol 131:181-186

Ferreira KN, Iverson TM, Maghlaoui K, Barber J, Iwata S (2004) Architecture of the photosynthetic oxygen-evolving center. Science 303:1831-1838

Fey H, Piano D, Horn R, Fischer D, Schmidt M, Ruf S, Schröder WP, Bock R, Büchel C (2008) Isolation of highly active photosystem II core complexes with a His-tagged Cyt b559 subunit from transplastomic tobacco plants. BBA 12:1501-1509

Kamiya N, Shen J-R (2003) Crystal structure of oxygen-evolving photosystem II from Thermosynechococcus vulcanus at 3.7- $\AA$ resolution. PNAS 100:98-103

Kiefersauer R, Than ME, Dobbek H, Gremer L, Melero M, Strobl S, Dias JM, Soulimane T, Huber R (2000) A novel free-mounting system for protein crystals: transformation and improvement of diffraction power by accurately controlled humidity changes. J Appl Cryst 33:1223-1230

Loll B, Kern J, Saenger W, Zouni A, Biesiadka J (2005) Towards complete cofactor arrangement in the $3.0 \AA$ resolution structure of photosystem II. Nature 438:1040-1044

Neuhoff V, Arold N, Taube D, Ehrhardt W (1988) Improved staining of proteins in polyacrylamide gels including isoelectric focusing gels with clear background at nanogram sensitivity using Coomassie Brillant Blue G-250 and R-250. Electrophoresis 9:255

Porra RJ, Thompson WA, Kriedmann PE (1989) Determination of accurate extinction coefficients and simultaneous equations for assaying chlorophylls a and b 98 with four different solvents: verifications of the concentration of chlorophyll standard by atomic absorption spectroscopy. BBA 975:384-394

Rhee KH, Morris EP, Zheleva D, Hankamer B, Kühlbrandt W, Barber J (1997) Two-dimensional structure of plant photosystem II at 8Å resolution. Nature 389:522-526

Smatanová IK, Gavira JA, Řezáčová P, Vácha F, García-Ruiz JM (2007) New techniques for membrane protein crystallization tested on Photosystem II core of Pisum sativum. Photosynth Res 90:255-259

Switzer R, Merril C, Shifrin S (1979) A highly sensitive silver stain for detecting proteins and peptides in polyacryamide gels. Anal Biochem 72:248 